\title{
NEPHROTIC SYNDROME steroid sensitive renal condition
}

By Maguzu Lulaga, (MD4, MUHAS- 2008/09)

\section{INTRODUCTION}

Nephrotic syndrome is primarily a paediatric disorder and is 15 times more common in children than adults. The incidence is $2-3 / 100,000$ children per year, and the majority of affected children will have steroidsensitive minimal change disease. The characteristic features of nephrotic syndrome are heavy proteinuria more than $40 \mathrm{mg} / \mathrm{m}^{2} / \mathrm{hr}$ in children, hypoalbuminemia $(<2.5 \mathrm{~g} / \mathrm{dL})$, edema, and hyperlipidemia ${ }^{[2]}$.

It may occur as a result of any glomerular disease and may rarely be associated with extrarenal conditions. The disease is categorized into three forms, idiopathic/ primary, secondary and congenital form. Among the three forms idiopathic form is the most common form in pediatrics ${ }^{[2,3]}$.

Most children (90\%) with nephrotic syndrome have a form of the idiopathic nephrotic syndrome. A wide variety of glomerular lesions can be seen in idiopathic nephritic syndrome. These include minimal change disease (85\%), mesangial proliferation (5\%), and focal segmental glomerulosclerosis (10\%). The remaining $10 \%$ of children with nephrotic syndrome have secondary nephrotic syndrome related to glomerular diseases such as membranous nephropathy (MN) or membranoproliferative glomerulonephritis (MPGN). Diseases such as sickle cell disease, diabetes mellitus, systemic lupus erythematosus, HIV/AIDS and syphilis can cause secondary nephrotic syndrome ${ }^{[2]}$.

\section{CASE DESCRIPTION}

A 4-year and 9 month-old female child referred from Bombo hospital (Tanga) presented with abdominal discomfort and generalized body swelling for 18 months. She was well till the age of 3 years when she suddenly experienced abdominal discomfort initially more severe around the umbilicus, non-radiating and later on generalised. This was a recurrent problem with an average of 1-2 months discomfort free time. It was not related to posture, eating or introduction of new food. There was also no history of diarrhea and vomiting during the time of illness.

During this time she also experienced generalized body swelling, which started around the eyes, face, neck and eventually it become generalized. There was decreased urine output in terms of amount and frequency and the urine colour was yellowish. There was no history of fever, micturition discomfort, syncope or dyspnoea during the illness. She also had no history of sting bite, trauma, cough or open Tuberculosis contact.

She was alert, afebrile $\left(37^{\circ} \mathrm{C}\right)$, with some palmar pallor, some finger clubbing, generalized pitting edema more marked on face and neck, normal breathing, there was nocyanosis, jaundice, lymph node enlargement, nose or ear discharge.

On cardiovascular system examination, the pulse rate was 80 beats/min which was regular of normal volume non-collapsing synchronous with the contralateral radial pulse, blood pressure was $100 / 70$, no areas of tenderness, the apex beat was in the $4^{\text {th }}$ intercostals space in the left mid-clavicular line, the first and second heart sounds were heard without abnormal sounds.

The abdomen was uniformly distended, moving normally with respiration, soft and non tender, no evidence of hepatosplenomegally and kidneys were not palpable, shifting dullness was positive, there were normal bowel sounds, the respiratory and central nervous systems were essentially normal.

Investigations requested were urinalysis, urine for culture and sensitivity, serum cholesterol and albumin, renal function test, Full Blood Picture and ESR, HIV antibody test and Esbach's test and a final diagnosis of nephrotic syndrome was reached. She was also found to have urinary tract infection.

She was given Prednisolone tablets $15 \mathrm{mg} 12$ hourly to continue with this for 6 months. Nitrofurantin tablets 
$25 \mathrm{mg} 12$ hourly for 14 days (for urinary tract infection). Salt was restricted in the patient's diet.

The patient was discharged home after 8 days and told to come on weekly basis for progress monitoring. The oedema had completely resolved by the sixth day of management. Urine protein decreased and the urine dipstick revealed protein $2++$ one day before discharge.

\section{DISCUSSION}

Results from the investigations done were characteristically in concordance with the four classical features of idiopathic nephrotic syndrome reported in literatures, massive proteinuria, hypoalbuminemia, oedema and hyperlipidemia especially in Minimal Change Disease (MCD).

The patient responded to predinisolone within 8 days of medication, this is a steroid sensitive case of nephrotic syndrome. Though it is still unknown what medications had been offered to her before coming to the hospital. The blood pressure was found to be $100 / 70 \mathrm{mmHg}$ this was done because patients with nephrotic syndrome may have hypertension primarily from the disease condition or secondarily as side effect of corticosteroid drugs or co-existence with nephritic syndrome.

The patient responded well to prednisolone since edema completely resolved by the eighth day of treatment. Urine protein decreased subsequently as evidenced by dipstick and Esbach's test done before and after start of prednisolone in order to monitor the progress.

\section{CONCLUSION AND RECOMMENDATIONS}

Nephrotic syndrome is a treatable condition and responds well with marked improvement within 1-2 weeks of predinisolone medication. Urine protein remains the standard measure of recovery. Regardless of histological type of nephrotic syndrome, proteinuria and body swelling seem to be the classical signs of nephrotic syndrome. High index of suspicion is important to differentiate between nephrotic and nephritic syndrome because studies have shown coexistence of the two syndromes in some patients.

\section{REFERENCES:}

1. Richard E., Md. Behrman (Editor), Robert M., Md. Kliegman (Editor), Hal B., Md. Jenson (Editor) By W B

2. Saunders; Nelson Textbook of Pediatrics 17th edition (May 2003):665

3. Graw MC ; Current Pediatric Diagnosis and Treatment $19^{\text {th }}$ edition. Hill Companies Inc.2009;659 